\title{
Un Corte de Aproximación para una Clase de Problemas de Programación Cuadrática Entera
}

\section{Edinson R. Montoro Alegre ${ }^{1}$ \& Martha Hilda Timoteo Sanchez $z^{2}$}

Resumen: En este trabajo se presenta un algoritmo para resolver una clase de Problemas de Programación Cuadrática Entera. El algoritmo resuelve una secuencia de a lo más $\sum_{j=1}^{n}\left(u_{j}-l_{j}\right)$ problemas de corte mínimo sobre un grafo con $n+2$ vértices donde $n$ es el número de variables en el problema.

Palabras clave: Programación entera, métodos de corte, problema de flujo y corte mínimo.

\section{An Aproximate Cut for a Class of. Problems of Entire Quadratic Programation}

\begin{abstract}
In this work is presented an algorithm that solve a class of integer quadratic program problems. The algorithm solves a secuence of at most $\sum_{j=1}^{n}\left(u_{j}-l_{j}\right)$ problems of minimum cut over a graph with $n+2$ vertex where $n$ is the number of variables in the problem.
\end{abstract}

Key words: Integer programation, cut method, flow problem and minimum cut.

\section{Introducción}

Consideremos el problema entero de la forma

$$
\begin{array}{r}
\min f(Y)=\sum_{j=1}^{n} \sum_{k=1}^{n} q_{j k} y_{j} y_{k}+\sum_{j=1}^{n} b_{j} y_{j} \\
\text { s.a } \quad l_{j} \leq y_{j} \leq u_{j} \quad j=1,2,3, \ldots, n
\end{array}
$$

donde $l_{j}$ y $u_{j}$ son enteros no negativos, $q_{j k}=q_{k j}, q_{j k} \leq 0$ para $j \neq k$ y $\sum_{j=1}^{n} q_{j k} \geq 0$ para $k=1,2,3, \ldots, n$.

Desarrollaremos un algoritmo el cual resuelve el (P0), resolviendo una secuencia de no más que $\sum u_{j}-l_{j}$ problemas de corte mínimo sobre un grafo con $n+2$ vértices.

Las ideas fundamentales para el algoritmo son: En el trabajo de Picard y Ratliff (ver [1]) "Minimum Cuts and Related Problem", una versión más simple del (P0) es tratada. En dicha versión cada variable $y_{j}$ sólo puede tomar como valor su cota inferior $l_{j}$ o $l_{j}+1$, y muestra que puede ser resuelto eficientemente como un problema de corte mínimo sobre un grafo. Si $Y^{*}$ es la solución óptima para este problema más simple, se mostrará que existe una solución óptima $Y^{0}$ para el problema original (P0) con $y_{j}^{0} \geq y_{j}^{*}$ para $j=1,2, \ldots, n$. Por lo tanto, para cada $j$ con

\footnotetext{
${ }^{1}$ UNMSMS, Facultad de Ciencias Matemáticas, e-mail: edinsonmontoro@yahoø.com

${ }^{2}$ UNMSMS, Facultad de Ciencias Matemáticas, e-mail: mtimoteos@yahoo.com
} 
$y_{j}^{*}=l_{j}+1$ le podemos incrementar a su cota inferior $l_{j}$ una unidad. Podemos entonces repetir este proceso hasta que obtengamos una solución óptima $Y^{*}$ con cada $y_{j}^{*}$ igual a su cota inferior. Mostraremos que tal solución es óptimo para el problema original. Antes de formular el algoritmo, necesitamos desarrollar el fundamento teórico.

\section{Resultados Teóricos}

Consideremos el problema

$$
\begin{aligned}
& \min f(X)=\sum_{j=1}^{n} \sum_{k=1}^{n} q_{j k} x_{j} x_{k}+\sum_{j=1}^{n} b_{j} x_{j} \\
& \text { s.a. } x_{j}=0,1,2, \ldots, u_{j}^{\prime}, \text { para } j=1,2,3, \ldots, n
\end{aligned}
$$

donde los $q_{j k}$ satisfacen las mismas condiciones que en (P0), y $u_{j}^{\prime}$ es un entero no negativo.

También consideremos el problema

$$
\begin{aligned}
& \min f(X)=\sum_{j=1}^{n} \sum_{k=1}^{n} q_{j k} x_{j} x_{k}+\sum_{j=1}^{n} \dot{b_{j}} x_{j} \\
& \text { s.a. } x_{j}=0 \text { o } 1, \text { para } j=1,2,3, \ldots, n
\end{aligned}
$$

Lemma 2.1. Si $X^{*}$ es una solución óptima para (P2), entonces existe una solución óptima $X^{\prime}$ $\operatorname{para}(P 1) \operatorname{con} x_{j}^{\prime} \geq x_{j}^{*}$

Prueba. Sea $X^{0}$ cualquier solución óptima para (P1) y sea

$$
R=\left\{j / x_{j}^{*}=1 \text { y } x_{j}^{0}=0\right\}
$$

Definimos entonces los vectores $X^{\prime}$ y $X^{\prime \prime}$ como

$$
\begin{aligned}
& x_{j}^{\prime}= \begin{cases}1 & , \text { si } j \in R \\
x_{j}^{0} & , \text { si } j \notin R\end{cases} \\
& x_{j}^{\prime \prime}= \begin{cases}0 & \text {, si } j \in R \\
x_{j}^{*} & , \text { si } j \notin R\end{cases}
\end{aligned}
$$

note que para cada $j \in R$ las variables $x_{j}^{*}=1$ y $x_{j}^{\prime \prime}=0$ (y para cada $j \notin R$, implica $x_{j}^{\prime \prime}=x_{j}^{*}$ ). Luego

$$
\begin{gathered}
f\left(X^{*}\right)=\sum_{j=1}^{n} \sum_{k=1}^{n} q_{j k} x_{j}^{*} x_{k}^{*}+\sum_{j=1}^{n} b_{j} x_{j}^{*} \\
f\left(X^{*}\right)=\sum_{j=1}^{n}\left(\sum_{k \in R} q_{j k} x_{j}^{*} x_{k}^{*}+\sum_{k \notin R} q_{j k} x_{j}^{*} x_{k}^{*}\right)+\sum_{j \in R} b_{j} x_{j}^{*}+\sum_{j \notin R} b_{j} x_{j}^{*} \\
f\left(X^{*}\right)=\sum_{j=1}^{n}\left(\sum_{k \in R} q_{j k} x_{j}^{*}+\sum_{k \notin R} q_{j k} x_{j}^{*} x_{k}^{*}\right)+\sum_{j \in R} b_{j}+\sum_{j \notin R} b_{j} x_{j}^{*} \\
f\left(X^{*}\right)=\sum_{j \in R} \sum_{k \in R} q_{j k} x_{j}^{*}+\sum_{j \in R} \sum_{k \notin R} q_{j k} x_{j}^{*} x_{k}^{*}+\sum_{j \notin R} \sum_{k \in R} q_{j k} x_{j}^{*}+\sum_{j \notin R} \sum_{k \notin R} q_{j k} x_{j}^{*} x_{k}^{*}+\sum_{j \in R} b_{j}+\sum_{j \notin R} b_{j} x_{j}^{*}
\end{gathered}
$$




$$
\begin{gathered}
f\left(X^{*}\right)=\sum_{j \in R} \sum_{k \in R} q_{j k}+\sum_{j \in R} \sum_{k \notin R} q_{j k} x_{k}^{*}+\sum_{j \notin R} \sum_{k \in R} q_{j k} x_{j}^{*}+\sum_{j \notin R} \sum_{k \notin R} q_{j k} x_{j}^{*} x_{k}^{*}+\sum_{j \in R} b_{j}+\sum_{j \notin R} b_{j} x_{j}^{*} \\
f\left(X^{*}\right)=\sum_{j \in R} \sum_{k \in R} q_{j k}+2 \sum_{j \in R} \sum_{k \notin R} q_{j k} x_{k}^{*}+\sum_{j \notin R} \sum_{k \notin R} q_{j k} x_{j}^{*} x_{k}^{*}+\sum_{j \in R} b_{j}+\sum_{j \notin R} b_{j} x_{j}^{*}
\end{gathered}
$$

Procediendo en forma similar:

$$
\begin{gathered}
f\left(X^{\prime \prime}\right)=\sum_{j=1}^{n} \sum_{k=1}^{n} q_{j k} x_{j}^{\prime \prime} x_{k}^{\prime \prime}+\sum_{j=1}^{n} b_{j} x_{j}^{\prime \prime} \\
f\left(X^{\prime \prime}\right)=\sum_{j=1}^{n}\left(\sum_{k \in R} q_{j k} x_{j}^{\prime \prime} x_{k}^{\prime \prime}+\sum_{k \notin R} q_{j k} x_{j}^{\prime \prime} x_{k}^{\prime \prime}\right)+\sum_{j \in R} b_{j} x_{j}^{\prime \prime}+\sum_{j \notin R} b_{j} x_{j}^{\prime \prime} \\
f\left(X^{\prime \prime}\right)=\sum_{j \in R} \sum_{k \notin R} q_{j k} x_{j}^{\prime \prime} x_{k}^{\prime \prime}+\sum_{j \notin R} \sum_{k \notin R} q_{j k} x_{j}^{\prime \prime} x_{k}^{\prime \prime}+\sum_{j \notin R} b_{j} x_{j}^{\prime \prime} \\
f\left(X^{\prime \prime}\right)=\sum_{j \notin R} \sum_{k \notin R} q_{j k} x_{j}^{\prime \prime} x_{k}^{\prime \prime}+\sum_{j \notin R} b_{j} x_{j}^{\prime \prime}
\end{gathered}
$$

luego

$$
f\left(X^{*}\right)-f\left(X^{\prime \prime}\right)=\sum_{j \in R} \sum_{k \in R} q_{j k}+2 \sum_{j \in R} \sum_{k \notin R} q_{j k} x_{k}^{*}+\sum_{j \in R} b_{j} \leq 0
$$

pues $X^{\prime \prime}$ es feasible para (P2) y $X^{*}$ es óptimo para (P2).

Por otro lado, notemos que para que cada $j \in R$ las variables $x_{j}^{0}=0$ y $x_{j}^{\prime}=1$ (y $x_{j}^{\prime}=x_{j}^{0}$ si $j \notin R$ ). Entonces, con un proceso de cálculo similar se obtiene

$$
f\left(X^{\prime}\right)-f\left(X^{0}\right)=\sum_{j \in R} \sum_{k \in R} q_{j k}+2 \sum_{j \in R} \sum_{k \notin R} q_{j k} x_{k}^{0}+\sum_{j \in R} b_{j}
$$

Luego, como $q_{j k} \leq 0$ para $j \neq k$ y por definición de $R, x_{j}^{0} \geq x_{j}^{*} \forall j \notin R$, entonces $q_{j k} x_{j}^{0} \leq q_{j k} x_{j}^{*}$, lo que implica

$$
2 \sum_{j \in R} \sum_{k \notin R} q_{j k} x_{j}^{0} \leq 2 \sum_{j \in R} \sum_{k \notin R} q_{j k} x_{k}^{*}
$$

Comparando (1) y (2) se tiene

$$
f\left(X^{\prime}\right)-f\left(X^{0}\right) \leq f\left(X^{*}\right)-f\left(X^{\prime \prime}\right) \leq 0
$$

de donde

$$
f\left(X^{\prime}\right) \leq f\left(X^{0}\right)
$$

y como $X^{\prime}$ es una solución feasible para (P1) y que satisface $x_{j}^{\prime} \geq x_{j}^{*}$, entonces $X^{\prime}$ es el óptimo de (P1) que satisface el lema.

Lemma 2.2. Si $X^{*}$ es una solución óptima de (P2) y $x_{j}^{*}=0$ para $j=1,2, \ldots, n$, entonces $X^{*}$ es el óptimo para (P1).

Prueba. Supongamos que $X^{*}$ no es óptimo, entonces existe un $X^{0}$ feasible para (P1) tal que $f\left(X^{0}\right)<f\left(X^{*}\right)=0$.

Definimos los vectores $X^{\prime}$ y $X^{\prime \prime}$ como

$$
x_{j}^{\prime}= \begin{cases}1 & , \text { si } x_{j}^{0} \geq 1 \\ 0 & , \text { si } x_{j}^{0}=0\end{cases}
$$




$$
x_{j}^{\prime \prime}=x_{j}^{0}-x_{j}^{\prime} \text { para } j=1,2, \ldots, n
$$

entonces

$$
\begin{gathered}
f\left(X^{0}\right)=f\left(X^{\prime}+X^{\prime \prime}\right)=\sum_{j=1}^{n} \sum_{k=1}^{n} q_{j k}\left(x_{j}^{\prime}+x_{j}^{\prime \prime}\right)\left(x_{j}^{\prime}+x_{j}^{\prime \prime}\right)+\sum_{j=1}^{n} b_{j}\left(x_{j}^{\prime}+x_{j}^{\prime \prime}\right) \\
f\left(X^{0}\right)=f\left(X^{\prime}\right)+f\left(X^{\prime \prime}\right)+2 \sum_{j=1}^{n} \sum_{k=1}^{n} q_{j k} x_{j}^{\prime} x_{j}^{\prime \prime}<0
\end{gathered}
$$

Como $X^{\prime}$ es feasible para (P2) y $X^{*}$ es óptimo para (P2) con $f\left(X^{*}\right)=0$, entonces se debe cumplir que $f\left(X^{\prime}\right) \geq 0$.

Por otro lado consideremos el término

$$
2 \sum_{j=1}^{n} \sum_{k=1}^{n} q_{j k} x_{j}^{\prime} x_{j}^{\prime \prime}
$$

De la hipótesis, tenemos que

$$
\sum_{k=1}^{n} q_{j k} \geq 0
$$

Si $x_{j}^{\prime}=1$, entonces

$$
\sum_{k=1}^{n} q_{j k} x_{j}^{\prime} \geq 0
$$

Además, $x_{j}^{\prime \prime}=0$ siempre que $x_{j}^{\prime}=0$, entonces

$$
2 \sum_{j=1}^{n} \sum_{k=1}^{n} q_{j k} x_{j}^{\prime} x_{j}^{\prime \prime} \geq 0
$$

Por tanto de (3) concluimos que $f\left(X^{\prime \prime}\right)<0$.

Si repetimos este proceso, cada vez haciendo $X^{0}=X^{\prime \prime}$, después de un número finito de veces debemos obtener un $X^{\prime \prime}$ con $f\left(X^{\prime \prime}\right)<0$ y $x_{j}^{\prime \prime}=0$ o 1 . Esto contradice el hecho de ser $X^{*}$ el óptimo de (P2).

Necesitamos hacer una observación más antes de comenzar con el algoritmo. Si un problema está en la forma (P0), debemos hacer el cambio de variable $x_{j}=y_{j}-l_{j}$ para obtener

$$
\begin{gathered}
\min f(X)+L=\sum_{j=1}^{n} \sum_{k=1}^{n} q_{j k} x_{j} x_{k}+\sum_{j=1}^{n} x_{j}\left(b_{j}+2 \sum_{k=1}^{n} l_{k} q_{j k}\right)+\sum_{j=1}^{n} \sum_{k=1}^{n} q_{j k} l_{j} l_{k} \\
\text { s.a. } x_{j}=0,1,2,3, \ldots, u_{j}-l_{j} .
\end{gathered}
$$

Como el término constante no afecta la optimización, (P3) es de la forma (P1). Notemos que los únicos coeficientes de (P3) que difieren de (P0) son aquellos que surgen del término lineal.

\section{Algoritmo:}

Paso 1. Dado un problema de la forma (P0), lo transformamos vía el cambio de variable $x_{j}=y_{j}-l_{j}$ a la forma $(\mathrm{P} 1)$.

Paso 2. Resolvemos el correspondiente problema (P2) como un problema de corte mínimo sobre un grafo para obtener una solución $X^{*}$. Sea $S=\left\{j / x_{j}^{*}=1\right\}$. 
Paso 3: Si $S=\emptyset$ paramos. Se sigue del lema 2 y de la transformación usada para obtener (P3) que $x_{j}=l_{j}$ para $j=1,2,3, \ldots, n$ es óptimo para $(\mathrm{P} 0)$.

Paso 4: Si $S \neq \emptyset$ hacemos $l_{j}=l_{j}+1$ para todo $j \in S$. Del lema 1 y de la transformación usada para obtener (P3), se sigue que la nueva $l_{j}$ es una cota inferior válida para $y_{j}$ en (P0). Para cada $j$ tal que $l_{j}=u_{j}$ permanentemente hacemos $y_{j}=l_{j}$. Volver al Paso 1 .

Desde que, al menos una cota inferior es incrementada en una unidad en cada iteración, está garantizado que el algoritmo terminará, en el peor de los casos después de $\sum_{j=1}^{n}\left(u_{j}-l_{j}\right)$ iteraciones.

\section{Ejemplo}

Consideremos el siguiente problema

$$
\begin{aligned}
& \min \left[\begin{array}{lll}
y_{1} & y_{2} & y_{3}
\end{array}\right]\left[\begin{array}{ccc}
6 & -4 & -2 \\
-4 & 8 & -4 \\
-2 & -4 & 20
\end{array}\right]\left[\begin{array}{l}
y_{1} \\
y_{2} \\
y_{3}
\end{array}\right]+\left[\begin{array}{lll}
y_{1} & y_{2} & y_{3}
\end{array}\right]\left[\begin{array}{lll}
-6 & -8 & -4
\end{array}\right] \\
& \text { s.a. } y_{1}=\{0,1,2,3\} \\
& y_{2}=\{0,1,2\} \\
& y_{3}=\{0,1,2,3,4\}
\end{aligned}
$$

Este problema ya está en la forma (P1). El correspondiente problema (P2) será

$$
\min \left[\begin{array}{lll}
y_{1} & y_{2} & y_{3}
\end{array}\right]\left[\begin{array}{ccc}
6 & -4 & -2 \\
-4 & 8 & -4 \\
-2 & -4 & 20
\end{array}\right]\left[\begin{array}{l}
y_{1} \\
y_{2} \\
y_{3}
\end{array}\right]+\left[\begin{array}{lll}
y_{1} & y_{2} & y_{3}
\end{array}\right]\left[\begin{array}{ccc}
-6 & -8 & -4
\end{array}\right]
$$

$$
\text { s.a. } \begin{aligned}
y_{1} & =\{0,1\} \\
& y_{2}=\{0,1\} \\
y_{3} & =\{0,1\}
\end{aligned}
$$

En orden a resolver el correspondiente problema (P2), podemos definir un grafo indireccionado con vértices $0,1,2,3, \ldots, n+1$ y capacidades definidas como sigue (ver [2] y [3])

$$
\begin{gathered}
c_{i j}=-q_{i j} \\
c_{j, n+1}=\operatorname{máx}\left\{\sum_{k=1}^{n} q_{j k}+b_{j}, 0\right\}, \\
c_{0 j}=\operatorname{máx}\left\{-\sum_{k=1}^{n} q_{j k}-b_{j}, 0\right\} .
\end{gathered}
$$

si $(S, \bar{S})$ es un corte de capacidad mínima en el grafo con $0 \in S$ y $n+1 \in \bar{S}$, una solución óptima para (P2) es $x_{j}=1$ para $j \in S$ y $x_{j}=0$ para $j \in \bar{S}$.

Para nuestro problema, el grafo generado es el siguiente 
cuyo corte mínimo es:

$$
\begin{aligned}
& y_{1}=1 \\
& y_{2}=1 \\
& y_{3}=0 .
\end{aligned}
$$

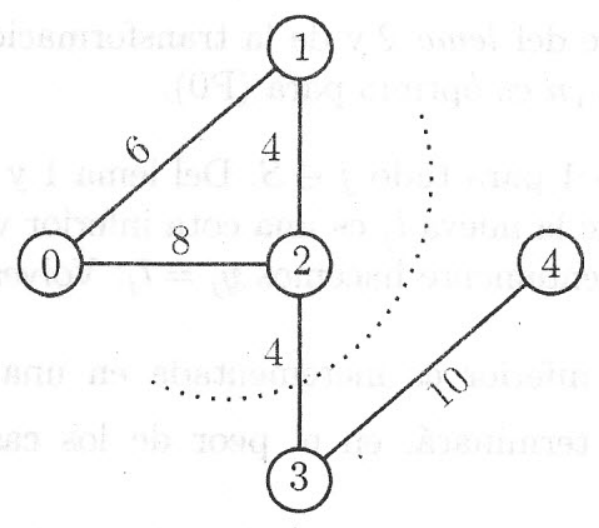

Entonces al problema se le incrementa $l_{1}=l_{1}+1, l_{2}=l_{2}+1, l_{3}=l_{3}$, y generamos otro problema

$$
\min \left[\begin{array}{lll}
y_{1} & y_{2} & y_{3}
\end{array}\right]\left[\begin{array}{ccc}
6 & -4 & -2 \\
-4 & 8 & -4 \\
-2 & -4 & 20
\end{array}\right]\left[\begin{array}{l}
y_{1} \\
y_{2} \\
y_{3}
\end{array}\right]+\left[\begin{array}{lll}
y_{1} & y_{2} & y_{3}
\end{array}\right]\left[\begin{array}{l}
-6 \\
-8 \\
-4
\end{array}\right]
$$

$$
\begin{array}{ll}
\text { s.a. } & y_{1}=\{1,2,3\} \\
& y_{2}=\{1,2\} \\
& y_{3}=\{0,1,2,3,4\}
\end{array}
$$

Este problema será el (P3) y no tiene la forma (P1), entonces hay que transformarlo haciendo el cambio de variable

$$
\begin{aligned}
& x_{1}=y_{1}-1 \\
& x_{2}=y_{2}-1 \\
& x_{3}=y_{3}
\end{aligned}
$$

lo reemplazamos en (I) y obtenemos

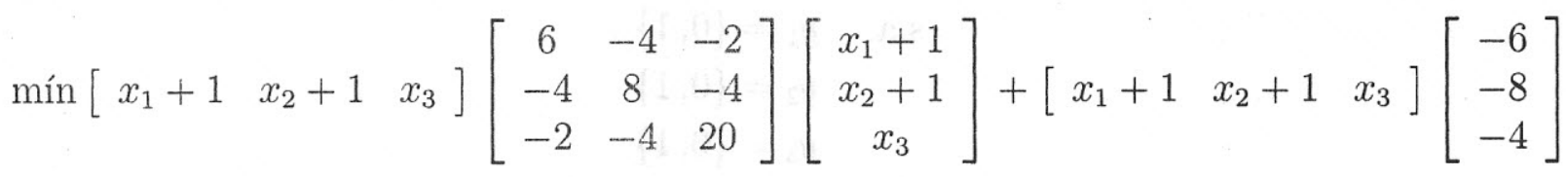

$$
\begin{aligned}
& \text { s.a. } \quad x_{1}=\{0,1,2\} \\
& x_{2}=\{0,1\} \\
& x_{3}=\{0,1,2,3,4\}
\end{aligned}
$$

operando:

$$
\begin{array}{r}
\left(\left[\begin{array}{lll}
x_{1} & x_{2} & x_{3}
\end{array}\right]+\left[\begin{array}{lll}
1 & 1 & 0
\end{array}\right]\right)\left[\begin{array}{ccc}
6 & -4 & -2 \\
-4 & 8 & -4 \\
-2 & -4 & 20
\end{array}\right]\left(\left[\begin{array}{l}
x_{1} \\
x_{2} \\
x_{3}
\end{array}\right]+\left[\begin{array}{l}
1 \\
1 \\
0
\end{array}\right]\right) \\
+\left(\left[\begin{array}{lll}
x_{1} & x_{2} & x_{3}
\end{array}\right]+\left[\begin{array}{lll}
1 & 1 & 0
\end{array}\right]\right)\left[\begin{array}{l}
-6 \\
-8 \\
-4
\end{array}\right]
\end{array}
$$




$$
\begin{array}{r}
{\left[\begin{array}{lll}
x_{1} & x_{2} & x_{3}
\end{array}\right]\left[\begin{array}{ccc}
6 & -4 & -2 \\
-4 & 8 & -4 \\
-2 & -4 & 20
\end{array}\right]\left[\begin{array}{l}
x_{1} \\
x_{2} \\
x_{3}
\end{array}\right]+\left[\begin{array}{lll}
x_{1} & x_{2} & x_{3}
\end{array}\right]\left[\begin{array}{ccc}
6 & -4 & -2 \\
-4 & 8 & -4 \\
-2 & -4 & 20
\end{array}\right]\left[\begin{array}{l}
1 \\
1 \\
0
\end{array}\right]} \\
+\left[\begin{array}{lll}
1 & 1 & 0
\end{array}\right]\left[\begin{array}{ccc}
6 & -4 & -2 \\
-4 & 8 & -4 \\
-2 & -4 & 20
\end{array}\right]\left[\begin{array}{l}
x_{1} \\
x_{2} \\
x_{3}
\end{array}\right]+\left[\begin{array}{lll}
1 & 1 & 0
\end{array}\right]\left[\begin{array}{ccc}
6 & -4 & -2 \\
-4 & 8 & -4 \\
-2 & -4 & 20
\end{array}\right]\left[\begin{array}{l}
1 \\
1 \\
0
\end{array}\right] \\
+\left[\begin{array}{lll}
x_{1} & x_{2} & x_{3}
\end{array}\right]\left[\begin{array}{l}
-6 \\
-8 \\
-4
\end{array}\right]+\left[\begin{array}{lll}
1 & 1 & 0
\end{array}\right]\left[\begin{array}{l}
-6 \\
-8 \\
-4
\end{array}\right]
\end{array}
$$

Por lo tanto

$$
\begin{aligned}
\min \left[\begin{array}{lll}
x_{1} & x_{2} & x_{3}
\end{array}\right]\left[\begin{array}{ccc}
6 & -4 & -2 \\
-4 & 8 & -4 \\
-2 & -4 & 20
\end{array}\right]\left[\begin{array}{l}
x_{1} \\
x_{2} \\
x_{3}
\end{array}\right]+\left[\begin{array}{lll}
x_{1} & x_{2} & x_{3}
\end{array}\right]\left[\begin{array}{c}
-2 \\
0 \\
-16
\end{array}\right] \\
\left.+\left(\left[\begin{array}{lll}
1 & 1 & 0
\end{array}\right]\left[\begin{array}{ccc}
6 & -4 & -2 \\
-4 & 8 & -4 \\
-2 & -4 & 20
\end{array}\right]\left[\begin{array}{l}
1 \\
1 \\
0
\end{array}\right]+\left[\begin{array}{c}
-6 \\
-8 \\
-4
\end{array}\right]\right]\right) \\
\text { s.a. } \quad x_{1}=\{0,1,2\} \\
x_{2}=\{0,1\} \\
x_{3}=\{0,1,2,3,4\}
\end{aligned}
$$

El correspondiente (P2) será

$$
\min \left[\begin{array}{lll}
x_{1} & x_{2} & x_{3}
\end{array}\right]\left[\begin{array}{ccc}
6 & -4 & -2 \\
-4 & 8 & -4 \\
-2 & -4 & 20
\end{array}\right]\left[\begin{array}{l}
x_{1} \\
x_{2} \\
x_{3}
\end{array}\right]+\left[\begin{array}{lll}
x_{1} & x_{2} & x_{3}
\end{array}\right]\left[\begin{array}{c}
-2 \\
0 \\
-16
\end{array}\right]
$$

s.a. $\quad x_{1}=\{0,1\}$

$$
x_{2}=\{0,1\}
$$$$
x_{3}=\{0,1,2\}
$$

El cual genera el siguiente grafo:

y el corte mínimo:

$x_{1}=1, x_{2}=1, x_{3}=0$.

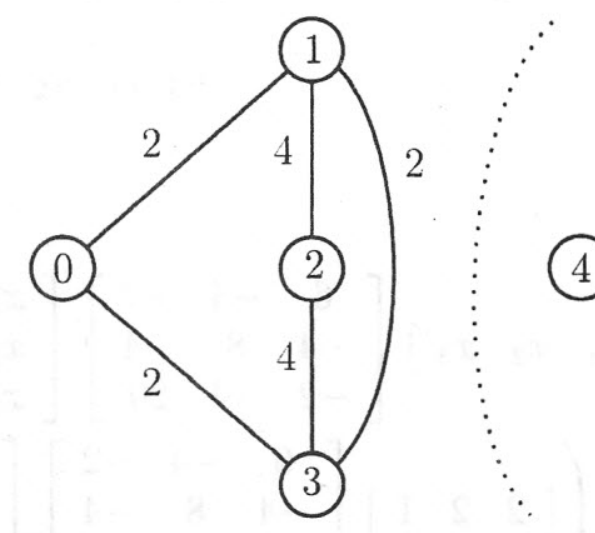

luego en (I) $y_{1}=2, y_{2}=2, y_{3}=1$.

Ahora en el problema original le aumentamos las cotas $l_{1}=l_{1}+1, l_{2}=l_{2}+1, l_{3}=l_{3}+1$. Luego

$$
\min \left[\begin{array}{lll}
y_{1} & y_{2} & y_{3}
\end{array}\right]\left[\begin{array}{ccc}
6 & -4 & -2 \\
-4 & 8 & -4 \\
-2 & -4 & 20
\end{array}\right]\left[\begin{array}{l}
y_{1} \\
y_{2} \\
y_{3}
\end{array}\right]+\left[\begin{array}{lll}
y_{1} & y_{2} & y_{3}
\end{array}\right]\left[\begin{array}{l}
-6 \\
-8 \\
-4
\end{array}\right]
$$


54 Un Corte de Aproximación para una Clase de Problemas de Programación Cuadrática Entera

$$
\begin{array}{ll}
\text { s.a. } & y_{1}=\{2,3\} \\
& y_{2}=\{2\} \\
& y_{3}=\{1,2,3,4\}
\end{array}
$$

será nuestro nuevo (P3), el cual no es de la forma (P1), entonces lo transformamos

$$
\begin{array}{ll}
x_{1}=\{0,1\} & x_{1}=y_{1}-2 \\
x_{2}=\{0\} & x_{2}=y_{2}-2 \\
x_{3}=\{0,1,2,3\} & x_{3}=y_{3}-1
\end{array}
$$

luego

$$
\begin{array}{r}
\min \left[\begin{array}{lll}
x_{1}+2 & x_{2}+2 & x_{3}+1
\end{array}\right]\left[\begin{array}{ccc}
6 & -4 & -2 \\
-4 & 8 & -4 \\
-2 & -4 & 20
\end{array}\right]\left[\begin{array}{l}
x_{1}+2 \\
x_{2}+2 \\
x_{3}+1
\end{array}\right] \\
+\left[\begin{array}{lll}
x_{1}+2 & x_{2}+2 & x_{3}+1
\end{array}\right]\left[\begin{array}{l}
-6 \\
-8 \\
-4
\end{array}\right]
\end{array}
$$

$$
\begin{array}{ll}
\text { s.a. } & x_{1}=\{0,1\} \\
& x_{2}=\{0\} \\
& x_{3}=\{0,1,2,3\}
\end{array}
$$

puede ser reescrito si consideramos

$$
\begin{array}{r}
{\left[\begin{array}{lll}
x_{1} & x_{2} & x_{3}
\end{array}\right]\left[\begin{array}{ccc}
6 & -4 & -2 \\
-4 & 8 & -4 \\
-2 & -4 & 20
\end{array}\right]\left[\begin{array}{l}
x_{1} \\
x_{2} \\
x_{3}
\end{array}\right]+\left[\begin{array}{lll}
x_{1} & x_{2} & x_{3}
\end{array}\right]\left[\begin{array}{ccc}
6 & -4 & -2 \\
-4 & 8 & -4 \\
-2 & -4 & 20
\end{array}\right]\left[\begin{array}{l}
2 \\
2 \\
1
\end{array}\right]} \\
+\left[\begin{array}{lll}
2 & 2 & 1
\end{array}\right]\left[\begin{array}{ccc}
6 & -4 & -2 \\
-4 & 8 & -4 \\
-2 & -4 & 20
\end{array}\right]\left[\begin{array}{l}
x_{1} \\
x_{2} \\
x_{3}
\end{array}\right]+\left[\begin{array}{lll}
2 & 2 & 1
\end{array}\right]\left[\begin{array}{ccc}
6 & -4 & -2 \\
-4 & 8 & -4 \\
-2 & -4 & 20
\end{array}\right]\left[\begin{array}{l}
2 \\
2 \\
1
\end{array}\right] \\
+\left[\begin{array}{lll}
x_{1} & x_{2} & x_{3}
\end{array}\right]\left[\begin{array}{l}
-6 \\
-8 \\
-4
\end{array}\right]+\left[\begin{array}{lll}
2 & 2 & 1
\end{array}\right]\left[\begin{array}{l}
-6 \\
-8 \\
-4
\end{array}\right]
\end{array}
$$

en

$$
\begin{aligned}
& \min \left[\begin{array}{lll}
x_{1} & x_{2} & x_{3}
\end{array}\right]\left[\begin{array}{ccc}
6 & -4 & -2 \\
-4 & 8 & -4 \\
-2 & -4 & 20
\end{array}\right]\left[\begin{array}{l}
x_{1} \\
x_{2} \\
x_{3}
\end{array}\right]+\left[\begin{array}{lll}
x_{1} & x_{2} & x_{3}
\end{array}\right]\left[\begin{array}{c}
-2 \\
0 \\
12
\end{array}\right] \\
& +\left(\left[\begin{array}{lll}
2 & 2 & 1
\end{array}\right]\left[\begin{array}{ccc}
6 & -4 & -2 \\
-4 & 8 & -4 \\
-2 & -4 & 20
\end{array}\right]\left[\begin{array}{l}
2 \\
2 \\
1
\end{array}\right]+\left[\begin{array}{lll}
2 & 2 & 1
\end{array}\right]\left[\begin{array}{l}
-6 \\
-8 \\
-4
\end{array}\right]\right) \\
& \text { s.a. } x_{1}=\{0,1\} \\
& x_{2}=\{0\} \\
& x_{3}=\{0,1,2,3\}
\end{aligned}
$$


y su correspondiente (P2)

$$
\begin{aligned}
\min \left[\begin{array}{lll}
x_{1} & x_{2} & x_{3}
\end{array}\right]\left[\begin{array}{ccc}
6 & -4 & -2 \\
-4 & 8 & -4 \\
-2 & -4 & 20
\end{array}\right]\left[\begin{array}{l}
x_{1} \\
x_{2} \\
x_{3}
\end{array}\right]+\left[\begin{array}{lll}
x_{1} & x_{2} & x_{3}
\end{array}\right]\left[\begin{array}{c}
-2 \\
0 \\
12
\end{array}\right] \\
\text { s.a. } \quad x_{1}=\{0,1\} \\
x_{2}=\{0\} \\
x_{3}=\{0,1\}
\end{aligned}
$$

y genera el grafo:

y el corte mínimo:

$x_{1}=0$

$x_{2}=0$

$x_{3}=0$

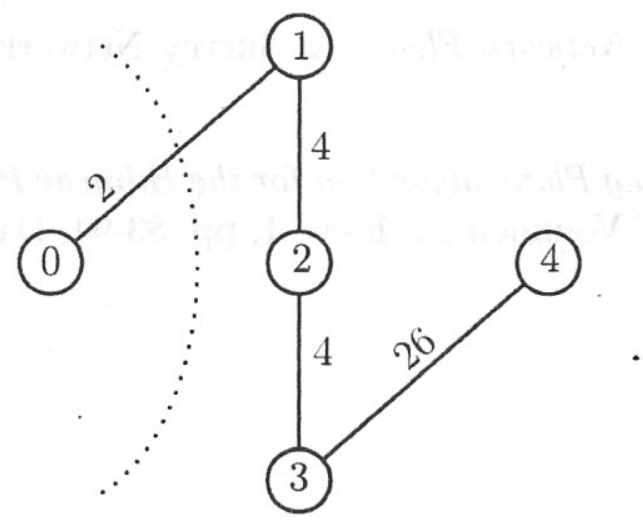

luego $X^{*}=(0,0,0)$ es óptimo para $(\mathrm{P} 1)$ por lema 2 y con el cambio de variable, produce ún óptimo para (P3) luego en (III) $y_{1}=2, y_{2}=2, y_{3}=1$ es la solución óptima para el problema original. 


\section{REFERENCIAS BIBLIOGRÁFICAS}

[1] J.C. Picard - H.D. Ratliff, Minimum cuts and related Problems, Network Volumen 2, Issue 1, Date March (1974).

[2] A.A. Assad, Multicommodity Network Flows, A. Survey Network Volumen 8, Issue 1, Date Spring, pp. 37-91 (1978).

[3] H. Vaish - C.M. Shelty, A cuting Plane algorithm for the Bilinear Programming Problem,Naval Renearch Logistics Quarterly, Volumen 24, Isswe 1, pp. 83-94, Date March (1977). 